\title{
Case Report Mechanical Thrombectomy as an Initial Treatment for Sinus Thrombosis
}

Yusuke Takahashi, Yoshitaka Suda, Ayana Saito, and Ryosei Wakasa

Objective: Sinus thrombosis often deteriorates despite anticoagulation therapy. Early endovascular therapy may lead to a better outcome in such cases. We report a case of sinus thrombosis treated by mechanical thrombectomy as an initial treatment.

Case Presentation: A 45-year-old female presented with headache and tonic seizure affecting the right arm. Computed tomography revealed subcortical hemorrhage at the right superior frontal gyrus. Occlusion of the superior sagittal sinus (SSS) was found on CTA and we diagnosed sinus thrombosis. Digital subtraction angiography demonstrated complete occlusion of the SSS and venous congestion at the middle third of the SSS. Immediately after diagnosis, we performed mechanical thrombectomy as an initial treatment. Partial recanalization was achieved after mechanical thrombectomy. Postoperative anticoagulation therapy was continued, and her symptom was improved.

Conclusion: Mechanical thrombectomy as an initial treatment for sinus thrombosis may be a useful treatment option when aggravation of the symptoms is predicted.

Keywords > sinus thrombosis, mechanical thrombectomy, initial treatment

\section{Introduction}

Cerebral sinus thrombosis is rare, with a reported incidence of 0.5 to $1 \%$ of all stroke patients. ${ }^{1)}$ Another study reported an incidence of 15.7/100000 persons/year. ${ }^{2)}$ The American Heart Association (AHA) guidelines recommend anticoagulant therapy and state that endovascular treatment should be considered for patients with symptom progression. ${ }^{3)}$ However, symptoms deteriorate in $24-25.8 \%$ of patients with cerebral sinus thrombosis, ${ }^{4,5)}$ and early endovascular treatment may improve the outcome in patients in whom symptoms are expected to deteriorate. ${ }^{5)}$ We report a patient with cerebral sinus thrombosis with

Department of Neurosurgery, Yuri-Kumiai General Hospital, Yuri-Honjo, Akita, Japan

Received: July 23, 2019; Accepted: October 19, 2019

Corresponding author: Yusuke Takahashi. Department of Neurosurgery, Yuri-Kumiai General Hospital, 38 Ie-Ushiro, Kawaguchi, Yuri-Honjo, Akita 015-8511, Japan

Email: s4001563@gmail.com

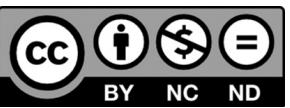

This work is licensed under a Creative Commons Attribution-NonCommercialNoDerivatives International License.

(C)2019 The Japanese Society for Neuroendovascular Therapy venous congestion for whom mechanical thrombectomy was performed on the day of arrival, leading to a favorable outcome.

\section{Case Presentation}

A 45-year-old female with the history of ulcerative colitis and uterine myoma treated with prednisolone and estradiol. She was admitted to the previous hospital with melena related to active ulcerative colitis. During admission, headache and dysesthesia of the right lower limb were noted, but computed tomography (CT) did not reveal any abnormality. She was discharged. Tonic seizure of the right upper limb developed 10 days after the onset of headache, persisting for 5 minutes. She was brought to our hospital by ambulance.

Physical examination on admission: The Japan Coma Scale score was 1 . There was no paralysis, but dysesthesia of the right lower limb was observed.

Hematology on admission: Laboratory data on admission were showed in Table 1. D-dimer was elevated to $3.3 \mu \mathrm{g} / \mathrm{mL}$. Imaging findings on admission: CT revealed subcortical hemorrhage involving a $1-\mathrm{cm}$ area in the right superior frontal gyrus (Fig. 1A). In the superior sagittal sinus (SSS), 
Table 1 Laboratory data on admission

\begin{tabular}{lcc} 
Variable & Reference range & On admission \\
White cell count $\left(\mathrm{per} \mathrm{mm}^{3}\right)$ & $3300-8600$ & 10000 \\
Hemoglobin $(\mathrm{g} / \mathrm{dL})$ & $11.6-14.8$ & 7.9 \\
Platelet count $\left(\mathrm{per} \mathrm{mm}^{3}\right)$ & $158,000-348,000$ & 408,000 \\
Urea nitrogen $(\mathrm{mg} / \mathrm{dL})$ & $8.0-20.0$ & 7.1 \\
Creatinine $(\mathrm{mg} / \mathrm{dL})$ & $0.46-0.79$ & 0.53 \\
Sodium $(\mathrm{mmol} / \mathrm{L})$ & $138-145$ & 139 \\
Potassium $(\mathrm{mmol} / \mathrm{L})$ & $3.6-4.8$ & 3.9 \\
Chloride $(\mathrm{mmol} / \mathrm{L})$ & $101-108$ & 108 \\
Prothrombin time international normalized ratio & $0.9-1.1$ & 0.97 \\
Activated partial thromboplastin time $(\mathrm{sec})$ & $24.0-32.0$ & 19.6 \\
D-dimer $(\mu \mathrm{g} / \mathrm{mL})$ & $0.0-1.0$ & 3.3 \\
\hline
\end{tabular}

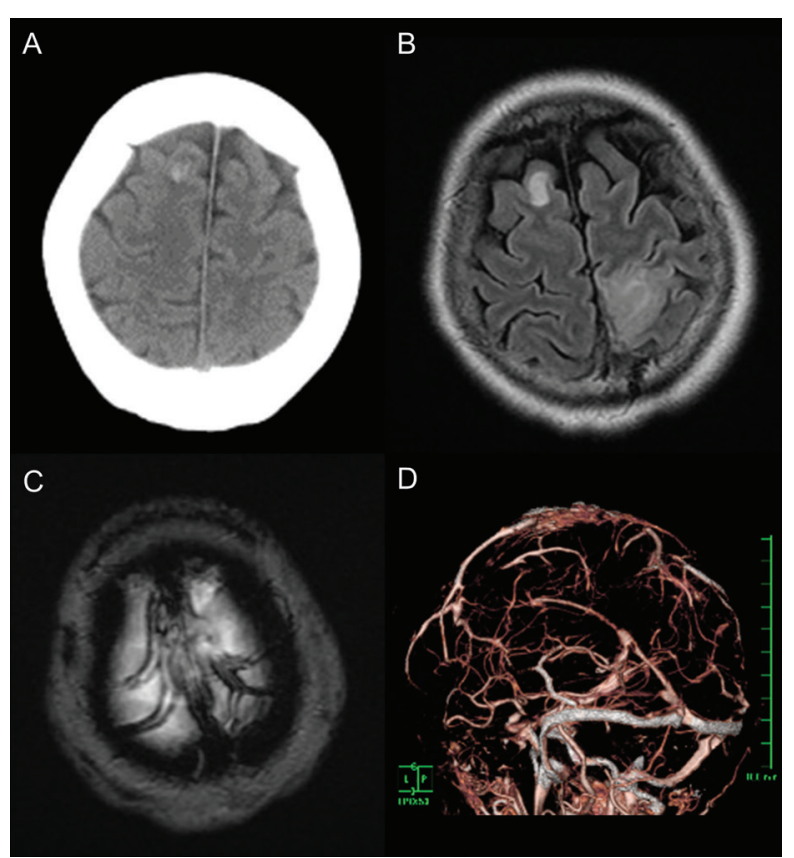

Fig. 1 Computed tomography showed suboccipital hemorrhage at the right superior frontal gyrus (arrow) (A). Fluid-attenuated inversion recovery imaging showed edema at the left postcentral gyrus (B). T2*-weighted imaging showed dilated cortical vein draining into the SSS, which suggested cortical vein thrombosis (C). Posterolateral view on 3D-CTA revealed occlusion of the SSS (D). SSS: superior sagittal sinus

there was no high-attenuation area. Magnetic resonance imaging using the FLAIR demonstrated high signal intensity in the left postcentral gyrus. T2*-weighted imaging revealed dilation of a cortical vein (Fig. 1B and 1C). On 3D-CTA, the SSS was not visualized, leading to a diagnosis of cerebral sinus thrombosis (Fig. 1D). In the venous phase of right internal carotid angiography, the SSS was not visualized, and a collateral pathway from the anterior/posterior regions of the SSS to the diploic vein was noted. However, there was no collateral pathway in the one-third middle area of the SSS, and prolongation of the capillary phase and venous congestion were observed. Furthermore, a contrast defect was present in the left transverse sinus, suggesting the presence of a thrombus (Fig. 2A and 2B).

\section{Treatment course}

As hemorrhage and venous congestion were observed during a 10-day course, endovascular treatment was performed in consideration of symptom deterioration. To approach the SSS, a route through the left internal jugular vein was selected for the following reasons: the degree of torsion of the left and right transverse and sigmoid sinuses was similar, and the presence of a thrombus in the left transverse sinus was suggested.

Heparin at 5000 units was administered. Subsequently, 1000 units of heparin were added at 1-hour intervals (total: 7000 units). For diagnosis, a 4-Fr JB2 (Medikit, Tokyo, Japan) was inserted into the right internal carotid artery, and a 6-Fr Shuttle (Cook Medical Inc., Bloomington, IN, USA) was inserted into the left internal jugular vein through the right femoral vein. A Penumbra 5MAX ACE 68 (Penumbra, Alameda, CA, USA) was guided to the middle third of the SSS using a Marksman (eV3 Covidien, Irvine, CA, USA) and CHIKAI-14 (Asahi Intecc Co., Ltd., Aichi, Japan) (Fig. 3A). Mechanical thrombectomy was performed using the direct aspiration first pass technique (ADAPT). A large amount of thrombi were collected from the Penumbra 5MAX ACE 68 and Shuttle. Recanalization of the SSS was not confirmed on imaging after the first thrombectomy session. In addition, two more thrombectomy sessions were conducted using ADAPT by inserting a Penumbra 5MAX ACE 68 into the middle third of the SSS (total: three sessions). After completion of the third session of thrombectomy, the posterior third of the SSS was visualized, revealing a thrombus adjacent to the confluence of the sinus (Fig. 3B). A Solitaire Platinum 


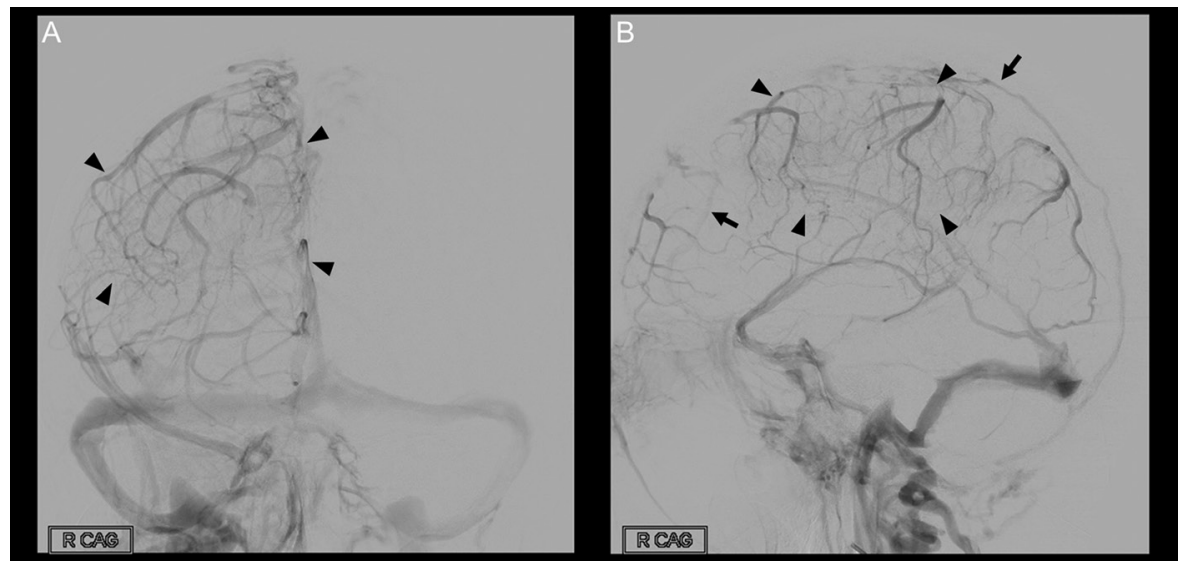

Fig. 2 Venous phase on anteroposterior (A) and lateral (B) views of right internal carotid artery angiography. The SSS was completely occluded. The anterior and posterior thirds of the cortical vein drained into the diploic vein (arrow), but the middle part of the cortical vein had no collateral route (arrowhead) and exhibited venous congestion. Filling defect was observed in the middle part of the left transverse sinus. SSS: superior sagittal sinus
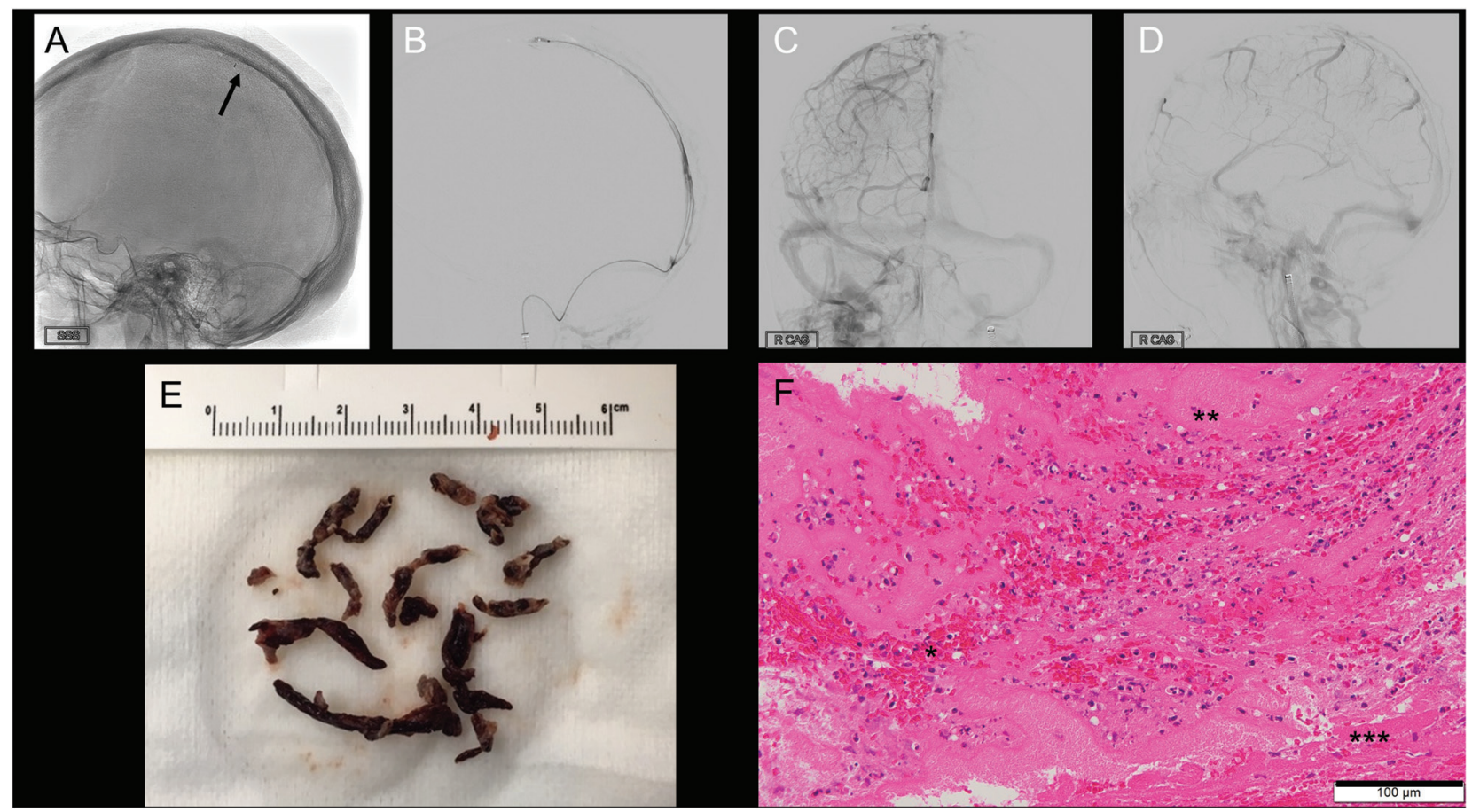

Fig. 3 Lateral view on right internal carotid artery angiography (A). A Penumbra 5MAX ACE 68 was placed at the middle part of the SSS (arrow). After three sessions of mechanical thrombectomy using the Penumbra system, injection demonstrated a filling defect at the posterior part of the superior straight sinus (arrow) (B). After thrombectomy using a Solitaire Platinum $6 \mathrm{~mm} \times 40 \mathrm{~mm}$ and Penumbra $5 \mathrm{MAX}$ ACE 68, partial recanalization of the posterior part of the SSS was archived (arrow) (C and D). After mechanical thrombectomy, a large amount of the clot was retrieved $(E)$. Hematoxylin-eosin staining of the retrieved thrombus $(F)(\times 400)$ showed red blood cells $\left({ }^{*}\right)$, fibrin-containing red blood cells $\left(^{* *}\right)$, and partially organized thrombus $\left.{ }^{(* *}\right)$. Scale bar $100 \mu \mathrm{m}$. SSS: superior sagittal sinus

$6 \times 40 \mathrm{~mm}$ (Medtronic, Minneapolis, MN, USA) was deployed and combined with a Penumbra 5MAX ACE 68 for thrombectomy. The middle third of the SSS was partially recanalized (Fig. 3C and 3D). We attempted to guide a Marksman to the anterior region of the SSS, but it was difficult. Thus, treatment was completed. A large amount of thrombi were collected through four sessions of thrombectomy (Fig. 3E). The thrombi were fresh, consisting of erythrocytes and fibrin. Their organization was advanced (Fig. 3F). After surgery, anticoagulant therapy with heparin (10,000 units/day) was continued. The Activated Partial Thromboplastin Time (APTT) the day after 


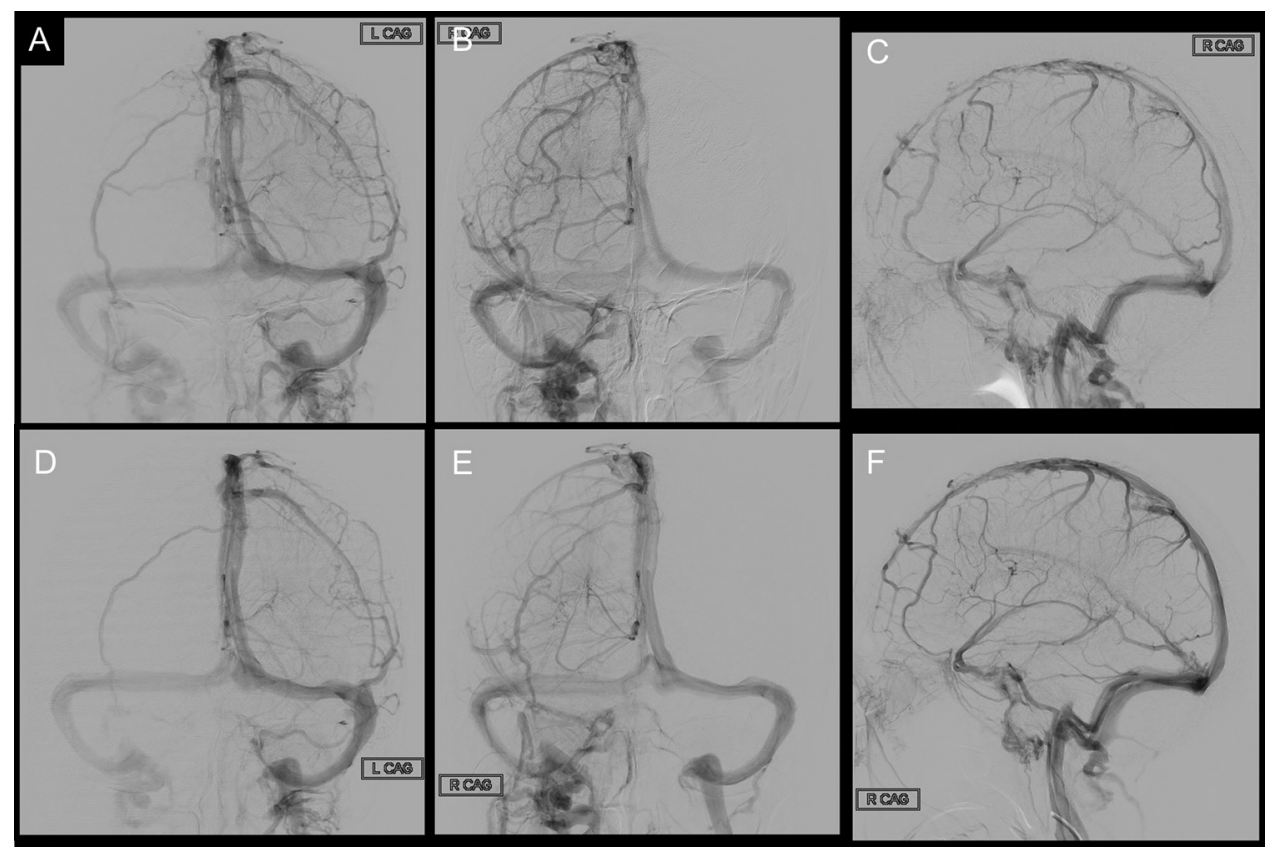

Fig. 4 Five days after mechanical thrombectomy, the anterior-posterior view on left carotid angiography (A), and anterior-posterior and lateral view on right carotid angiography (B and $\mathbf{C}$ ) showed partial recanalization of the anterior part of the SSS. One month after mechanical thrombectomy, the anteriorposterior view on left carotid angiography (D), and anterior-posterior and lateral view of the right carotid angiography ( $E$ and $\mathbf{F}$ ) showed complete recanalization of the SSS. SSS: superior sagittal sinus

surgery was 21.4 seconds, but atypical genital bleeding persisted. Heparin was switched to edoxaban at $30 \mathrm{mg}$ the day after surgery, although this agent should not be indicated for this condition. Furthermore, the administration of levetiracetam at $1000 \mathrm{mg} /$ day was started to prevent seizure. After surgery, her consciousness became clear and dysesthesia of the right lower limb decreased. Cerebral angiography 5 days after surgery confirmed recanalization of the SSS involving the anterior region, but the thrombus remained (Fig. 4A-4C). The patient was discharged 13 days after surgery with a modified Rankin Scale (mRS) score of 0. A postoperative blood test demonstrated normal protein $\mathrm{S}$, protein $\mathrm{C}$, and anti-phospholipid antibody levels. On cerebral angiography one month after treatment, the thrombus was not visualized and complete recanalization of the SSS was confirmed (Fig. 4D-4F). During the 3-month postoperative follow-up, the course has been favorable under therapy with edoxaban at 30 $\mathrm{mg}$ and levetiracetam at $1000 \mathrm{mg} /$ day.

\section{Discussion}

We report a patient for whom mechanical thrombectomy was performed as the initial treatment for cerebral sinus thrombosis. This disorder is relatively rare, and its reported incidence is $0.5-1 \%$ of all stroke patients. ${ }^{1)}$ Another study reported an incidence of 15.7/100,000 persons/year. ${ }^{2)}$ This disorder frequently develops in young to middle-aged persons with underlying diseases. Etiological factors include pregnancy/puerperium, the use of oral contraceptives, antithrombin III deficiency, protein $\mathrm{C} / \mathrm{S}$ deficiency, anti-phospholipid antibody syndrome, head trauma, collagen disease, malignant diseases, and hematological diseases. Inflammatory bowel diseases are involved in the onset of cerebral sinus thrombosis in $1.6 \%$ of all patients with this disorder. ${ }^{3)}$ In a Japanese study, cerebral sinus thrombosis developed in $2.2 \%$ of patients with ulcerative colitis. ${ }^{6}$ Furthermore, another study found that cerebral sinus thrombosis frequently develops in the active phase of ulcerative colitis. ${ }^{7)}$ In the present case, the oral administration of prednisolone and estradiol was a background factor, and active ulcerative colitis may have caused cerebral sinus thrombosis. Concerning treatment, two previous randomized comparative studies demonstrated the usefulness of the intravenous injection of heparin and subcutaneous injection of low-molecular-weight heparin. $\left.{ }^{89}\right)$ The AHA guidelines in 2011 recommended anticoagulant therapy as the first-choice treatment. ${ }^{3)}$ On the other hand, anticoagulant therapy leads to an unfavorable outcome in some patients. According to the International Study on Cerebral 
Vein and Dural Sinus Thrombosis in 2004, anticoagulant therapy was performed for $83.3 \%$ of the subjects, and the mRS score was 0 in $57.1 \%$, whereas it was $3-6$ in $13.4 \%$, resulting in an unfavorable or fatal outcome. ${ }^{10)}$ The study suggested that an age of $\geq 37$ years, male sex, coma, psychiatric diseases, presence of cerebral hemorrhage on CT at the time of admission, thrombosis of the deep venous system, central nervous infection, and malignant tumors are risk factors for an unfavorable outcome. In the present case, two risk factors: an age of $\geq 37$ years and the presence of cerebral hemorrhage were present.

Regarding the progression of cerebral sinus thrombosis, Bushnaq et al. reported symptom deterioration in 38 of 147 patients, and suggested that the number of affected sinuses, consciousness level on admission (Glasgow Coma Scale: $<15$ ), a platelet count of $\leq 225,000$, blood sodium level of $\leq 139$, epilepsy at the time of onset, oral contraceptive use and congestive papillae are risk factors for symptom deterioration. ${ }^{5}$ Sheth et al. classified affected sinus venous return into three patterns: (1) a vein-visualization-free pattern, (2) a pattern in which a vein is visualized, but it does not function as a collateral pathway, and (3) a pattern in which a vein that functions as a collateral pathway is present. They reported symptom deterioration in 10 of 13 patients with cerebral sinus thrombosis in whom there was no vein functioning as a collateral pathway. ${ }^{11)}$ Thus, endovascular treatment early after diagnosis may promptly result in the resumption of venous return in high-risk patients, thereby preventing symptom deterioration. In the present case, the entire length of the SSS was affected by thrombi. In the anterior and posterior areas, diploic-veinmediated collateral pathways were observed, but there was no collateral pathway in the middle third. A delay in the capillary phase and venous congestion were noted. Based on the pattern of venous return, our patient belonged to the high-risk group reported by Sheth et al. Furthermore, a reduction in the consciousness level, epilepsy at the time of onset, and the use of an oral contraceptive, as described by Bushnaq et al., were present. If anticoagulant therapy alone had been performed, the risk of symptom deterioration would have been high. Therefore, early endovascular treatment may have been appropriate.

As endovascular treatment procedures, thrombolysis with urokinase, ${ }^{12)}$ crushing with a balloon, ${ }^{13)}$ mechanical thrombectomy with an AngioJet (Boston Scientific, Natick, MA, USA) ${ }^{14)}$ and stenting ${ }^{15)}$ have been reported. Concerning thrombectomy with a stent retriever and Penumbra system, as adopted in the present case, Sticzen et al. used a
Trevo XP (Stryker Neurovascular, Fremont, CA, USA) alone or in combination with a Penumbra system to treat cerebral sinus thrombosis in 13 non-responders to anticoagulant therapy, and reported successful recanalization in 12 of the 13 patients, including 8 in whom partial recanalization was achieved, and that the outcome was favorable in 12 , with an mRS score of $\geq 2 .{ }^{16)}$ According to a systematic review published by Ilyas et al. who conducted endovascular treatment for cerebral sinus thrombosis, the complete recanalization rate was $69.0 \%$ and marked hemorrhage was noted in $8.7 \%$. In addition, the mRS score was $\geq 2$ in $76 \%$, reflecting a favorable outcome. Of these patients, a stent retriever was used for $11.5 \%$ and a Penumbra system for $8.9 \%$. There were no significant differences in the recanalization or hemorrhage rates between the group in which a stent retriever or Penumbra system was used and the group in which other devices were used. ${ }^{17)}$ However, balloon and rheolytic catheters are hard and large. Siddiqui et al. reported that rheolytic catheters were associated with a low recanalization rate and perioperative complications. ${ }^{18)}$ The stent retriever and Penumbra system that we used have favorable guiding properties, and they may increase the recanalization rate, thereby reducing the incidence of perioperative complications.

According to the above systematic review, the interval from onset until endovascular treatment was $\geq 1$ day in 72 patients and $\leq 24$ hours in 6 . Patients who underwent endovascular treatment within 24 hours after onset (early treatment group) had significantly poorer outcomes. However, as many studies performed endovascular treatment for severe-status patients or for those with symptom deterioration, we cannot exclude the possibility that many severe-status patients were included in the early treatment group. Dashti et al. conducted thrombectomy with an AngioJet as initial treatment for 13 patients with cerebral sinus thrombosis, and reported favorable outcomes in $10 .{ }^{19}$

In the present case, partial recanalization was achieved after thrombectomy. Ilyas et al. reported no significant difference in the outcome between complete and partial recanalization groups. Even if partial recanalization is achieved, sufficient anticoagulant therapy may increase the number of thrombi exposed to an anticoagulant, leading to therapeutic effects.

Furthermore, in the present case, histopathological examination revealed that the thrombus consisted of erythrocytes and fibrin. However, fibrin organization was partially advanced. A previous study found that the recanalization rate for thrombi containing a large amount of fibrin was 
lower than that for those consisting of erythrocytes after thrombectomy for intracranial arterial occlusion. ${ }^{20)}$ In the present case, organized fibrin was present, and anticoagulant therapy alone may not have led to complete recanalization. Moreover, elective treatment may promote thrombus organization, making thrombectomy difficult. From this viewpoint, early thrombectomy may be useful.

\section{Conclusion}

We report a patient for whom endovascular treatment was performed as the initial treatment for cerebral sinus thrombosis. Some patients with cerebral sinus thrombosis do not respond to anticoagulant therapy, and early venous return resumption by endovascular treatment may prevent symptom deterioration.

\section{Acknowledgments}

We thank Drs. Sugita and Yamauchi (Department of Pathological Diagnosis, Yuri Kumiai General Hospital) for their help in the preparation of pathological specimens.

\section{Disclosure Statement}

We declare no conflict of interest.

\section{References}

1) Stam J: Thrombosis of the cerebral veins and sinuses. N Engl J Med 2005; 352: 1791-1798.

2) Devasagayam $S$, Wyatt $B$, Leyden $J$, et al: Cerebral venous sinus thrombosis incidence is higher than previously thought: a retrospective population-based study. Stroke 2016; 47: 2180-2182.

3) Saposnik G, Barinagarrementeria F, Brown RD, et al: Diagnosis and management of cerebral venous thrombosis: a statement for healthcare professionals from the American Heart Association/American Stroke Association. Stroke 2011; 42: 1158-1192.

4) Bousser MG, Ferro JM: Cerebral venous thrombosis: an update. Lancet Neurol 2007; 6: 162-170.

5) Bushnaq SA, Qeadan F, Thacker T, et al: High-risk features of delayed clinical progression in cerebral venous thrombosis: a proposed prediction score for early intervention. Interv Neurol 2018; 7: 297-307.

6) Ando K, Fujiya M, Nomura $Y$, et al: The incidence and risk factors of venous thromboembolism in Japanese inpatients with inflammatory bowel disease: a retrospective cohort study. Intest Res 2018; 16: 416-425.
7) Katsanos $\mathrm{AH}$, Katsanos KH, Kosmidou M, et al: Cerebral sinus venous thrombosis in inflammatory bowel diseases. QJM 2013; 106: 401-413.

8) Einhaupl KM, Villringer A, Meister W, et al. Heparin treatment in sinus venous thrombosis. Lancet (London, England) 1991; 338: 597-600.

9) de Bruijn SF, Stam J: Randomized, placebo-controlled trial of anticoagulant treatment with low-molecular-weight heparin for cerebral sinus thrombosis. Stroke 1999; 30: 484- 488.

10) Ferro JM, Canhão P, Stam J, et al: Prognosis of cerebral vein and dural sinus thrombosis: results of the International Study on Cerebral Vein and Dural Sinus Thrombosis (ISCVT). Stroke 2004; 35: 664-670.

11) Sheth SA, Trieu H, Liebeskind DS, et al: Venous collateral drainage patterns predict clinical worsening in dural venous sinus thrombosis. J Neurointerv Surg 2018; 10: 171-175.

12) Canhão $P$, Falcão F, Ferro JM: Thrombolytics for cerebral sinus thrombosis: a systematic review. Cerebrovasc Dis 2003; 15: 159-166.

13) Chaloupka JC, Mangla S, Huddle DC. Use of mechanical thrombolysis via microballoon percutaneous transluminal angioplasty for the treatment of acute dural sinus thrombosis: case presentation and technical report. Neurosurgery 1999; 45: 650-656; discussion 656-657.

14) Curtin KR, Shaibani A, Resnick SA, et al: Rheolytic catheter thrombectomy, balloon angioplasty, and direct recombinant tissue plasminogen activator thrombolysis of dural sinus thrombosis with preexisting hemorrhagic infarctions. AJNR Am J Neuroradiol 2004; 25: 1807-1811.

15) Adachi $H$, Mineharu $Y$, Ishikawa $T$, et al: Stenting for acute cerebral venous sinus thrombosis in the superior sagittal sinus. Interv Neuroradiol 2015; 21: 719-723.

16) Styczen H, Tsogkas I, Liman J, et al: Endovascular mechanical thrombectomy for cerebral venous sinus thrombosis: a single-center experience. World Neurosurg 2019; 127: e1097-e1103.

17) Ilyas A, Chen CJ, Raper DM, et al: Endovascular mechanical thrombectomy for cerebral venous sinus thrombosis: a systematic review. J Neurointerv Surg 2017; 9: 1086-1092.

18) Siddiqui FM, Dandapat S, Banerjee C, et al: Mechanical thrombectomy in cerebral venous thrombosis: systematic review of 185 cases. Stroke 2015; 46: 1263-1268.

19) Dashti SR, Hu YC, Yao T, et al: Mechanical thrombectomy as first-line treatment for venous sinus thrombosis: technical considerations and preliminary results using the AngioJet device. J Neurointerv Surg 2013; 5: 49-53.

20) Shin JW, Jeong HS, Kwon HJ, et al: High red blood cell composition in clots is associated with successful recanalization during intra-arterial thrombectomy. PLOS ONE 2018; 13: e0197492. 